\title{
SOME REMARKS \\ ON EIGENFUNCTION EXPANSIONS FOR SCHRÖDINGER OPERATORS WITH NON-LOCAL POTENTIALS
}

\author{
ARNE JENSEN
}

We consider two-body Schrödinger operators $H=-\Delta+V$ in $L^{2}\left(\mathrm{R}^{3}\right)$ with a not necessarily local potential $V$ that decreases faster than $|x|^{-1-\varepsilon}$ at infinity. We prove the existence of two families $\varphi_{ \pm}(x, \xi)$ of generalized eigenfunctions of $H$ such that the generalized Fourier transforms

$$
\left(\mathscr{F}_{ \pm} f\right)(\xi)=\lim _{N \rightarrow \infty} \int_{|x|<N} f(x) \overline{\varphi_{ \pm}(x, \xi)} d x
$$

are unitary maps from the subspace of absolute continuity for $H$ onto $L^{2}\left(\mathbf{R}^{3}\right)$. Let $H_{\mathrm{ac}}$ denote the absolutely continuous part of $H$. Then $H_{\mathrm{ac}}=\mathscr{F}_{ \pm}^{*} M_{|\cdot|^{2}} \mathscr{F}_{ \pm}$where $M_{|\cdot|^{2}}$ denotes multiplication with $|\xi|^{2}$. Using the results of Kuroda [4] we establish the connection with scattering theory and prove a rigorous version of the formal connection between the scattering matrix and the generalized eigenfunctions used in physics.

For $V$ multiplication by a real-valued function the above results have been proved by Agmon [2]. We prove the results for a general $V$ by constructing the generalized eigenfunctions using Sobolev's lemma. This method is due to Agmon [1] and has been used by Yamada [8] for constructing eigenfunctions for the Dirac operator with a multiplicative potential. The applicability of the method depends on the fact that we restrict our attention to the physically relevant 3dimensional case. For a general $V$ the regularity in the $x$-variable is weaker than the one obtained in [2] for multiplicative $V$.

\section{Definitions, notations and assumptions on $V$.}

We denote by $L^{2}$ the space $L^{2}\left(\mathrm{R}^{3}\right)$, the norm $\|\cdot\|$ and the inner product $(\cdot, \cdot), \Omega$ denotes the unit sphere in $\mathrm{R}^{3}$ and $L^{2}(\Omega)$ the space of square integrable functions with respect to the surface measure on $\Omega$. The norm and the inner product are denoted $\|\cdot\|_{\Omega}$ and $(\cdot, \cdot)_{\Omega}$. For $s \in \mathrm{R}, L^{2, s}=H^{0, s}$ denotes the weighted $L^{2}$ space

Received January 24, 1977. 


$$
L^{2, s}=\left\{f \mid\left(1+|x|^{2}\right)^{s / 2} f(x) \in L^{2}\right\}
$$

with the norm

$$
\|f\|_{0, s}=\left\|\left(1+|x|^{2}\right)^{s / 2} f\right\| .
$$

$\mathscr{F}\left(\mathscr{F}^{-1}\right)$ denotes the (inverse) Fourier transform on the temperate distributions in $\mathrm{R}^{3}$. The Sobolev space $H^{m}=H^{m, 0}, m \in \mathrm{R}$, is the Hilbert space of temperate distributions given by

$$
H^{m}=\left\{f \mid \mathscr{F} f \in L^{2, m}\right\}
$$

with the norm

$$
\|f\|_{m, 0}=\|\mathscr{F} f\|_{0, m} .
$$

The weighted Sobolev space $H^{m, s}$ is defined for $m, s \in \mathrm{R}$ by

$$
H^{m, s}=\left\{f \mid\left(1+|x|^{2}\right)^{s / 2} f \in H^{m}\right\}
$$

with the norm

$$
\|f\|_{m, s}=\left\|\left(1+|x|^{2}\right)^{s / 2} f\right\|_{m, 0} .
$$

The dual of $H^{m, s}$ is identified with $H^{-m,-s}$ and the duality is written

$$
(f, g)=\int_{R^{3}} \overline{f(x)} g(x) d x .
$$

For two Hilbert spaces $K$ and $L \quad \mathscr{B}(K, L)$ denotes the bounded linear operators from $K$ to $L$, equipped with the operator norm. The adjoint of an operator $T$ is denoted $T^{*}$. The subspace of compact operators is denoted $\mathscr{C}(K, L)$.

For any $0<\theta<1$ and $s \in \mathrm{R}$ we denote by $C^{\theta, s}$ the continuous functions on $\mathrm{R}^{3}$ such that $\left(1+|x|^{2}\right)^{s / 2} f \in L^{\infty}\left(R^{3}\right)$ and such that $\left(1+|x|^{2}\right)^{s / 2} f(x)$ satisfies a uniform Hölder condition of order $\theta . C^{\theta, s}$ is a Banach space with the norm

$|f|_{\theta, s}=\sup _{x \in R^{3}}\left(1+|x|^{2}\right)^{s / 2}|f(x)|+\sup _{\substack{x, y \\ 0<|x-y|<1}}\left[\left(1+|x|^{2}\right)^{s / 2} \frac{|f(x)-f(y)|}{|x-y|^{\theta}}\right]$.

$H_{0}$ denotes the Laplacian $-\Delta$ in $L^{2}$ with domain $D\left(H_{0}\right)=H^{2,0}$.

Assumptions on $V$. Let $V$ be a closed symmetric operator on $L^{2}$ with $D(V) \supseteqq D\left(H_{0}\right)$ such that for some $s>\frac{1}{2}$

$$
V \in \mathscr{C}\left(H^{2,0}, H^{0,2 s}\right),
$$

and such that $V$ has an extension to $H^{2,-s}$, also denoted $V$, with the property

$$
V \in \mathscr{C}\left(H^{2,-s}, H^{0, s}\right) \text {. }
$$


Note. In the remainder of this paper $s$ denotes the constant introduced above.

Remarks (i) When $V$ is multiplication by a real-valued function $v(x)$ condition (1.1) is Agmon's condition (SR), [2]. In this case (1.2) follows from (1.1). A sufficient condition on $v$ is

$$
\sup _{x \in \mathrm{R}^{3}}\left[(1+|x|)^{2+2 \varepsilon} \int_{|x-y|<1}|v(y)|^{2}|x-y|^{1-2 \theta} d y\right]<\infty
$$

for some $\varepsilon>0$ and $0<\theta<\frac{1}{2}$. The results in [2] are proved under this assumption.

(ii) It follows from [3] that (1.3) is also necessary for $v$ to satisfy (1.1). (This remark is due to E. Balslev).

(iii) If $\frac{1}{2}<s^{\prime} \leqq s$ then (1.1) and (1.2) are satisfied with $s^{\prime}$ instead of $s$.

(iv) Under (1.1) and (1.2) the results of [4] hold.

From the assumptions on $V$ and the Kato-Rellich theorem follow that by $H=H_{0}+V, D(H)=D\left(H_{0}\right)$ is defined a selfadjoint operator on $L^{2}$.

We denote by $\gamma(k), k>0$, the trace operator defined on $C_{0}^{\infty}\left(\mathbf{R}^{3}\right)$ by (using polar coordinates $x=k \omega, k>0, \omega \in \Omega)(\gamma(k) f)(\cdot)=f(k) \cdot \gamma(k)$ has an extension to a bounded linear operator $\gamma(k): H^{t, 0} \rightarrow L^{2}(\Omega)$ for any $t>\frac{1}{2}$. There exists a constant $C$ such that

$$
\|\gamma(k)\| \leqq C \quad \text { for all } k>0,
$$

and if $t<\frac{3}{2}$ there exists a constant $C^{\prime}$ such that

$$
\left\|\gamma\left(k_{1}\right)-\gamma\left(k_{2}\right)\right\| \leqq C^{\prime}\left|k_{1}-k_{2}\right|^{t-\frac{1}{2}} \text { for all } k_{1}, k_{2}>0 .
$$

The norms in (1.4) and (1.5) are the operator norm on $\mathscr{B}\left(H^{t, 0}, L^{2}(\Omega)\right)$. For a proof of these results, see [5 p. 44].

\section{Some lemmas.}

We state without proof some results which we need in section 3 and 4 . The following result is known as Sobolev's lemma. For a proof, see [7].

LEMMA 2.1. For $t>\frac{3}{2}, u \in H^{t, 0}$ is a bounded continuous function (after correction on a null set) such that $|u(x)| \leqq C\|u\|_{t, 0}$ for some $C>0$. For any $\theta, 0<\theta<t-\frac{3}{2}, \theta<1$, there exists a constant $C^{\prime}$ such that

$$
|u(x)-u(y)| \leqq C^{\prime}\|u\|_{t, 0}|x-y|^{\theta} .
$$

We denote by $e_{+}(H)$ the discrete set of positive eigenvalues of $H$. (It is proved in [4] that $e_{+}(H)$ is discrete under our assumptions on $V$ ). The resolvent is denoted $R(z)=(H-z)^{-1}$. 
Lemma 2.2. For any $t>\frac{1}{2}$ and $\lambda \in R_{+} \backslash e_{+}(H)$ the following limits exist in the operator norm on $\mathscr{B}\left(H^{0, t}, H^{2,-t}\right)$

$$
R(\lambda \pm i 0)=\lim _{\varepsilon \downarrow 0} \mathrm{R}(\lambda \pm i \varepsilon)
$$

The convergence is uniform on compact subsets of $R_{+} \backslash e_{+}(H)$.

This result is due to Agmon [2] for multiplicative $V$. It is proved under our assumptions on $V$ in [4].

We define $\varphi_{\delta}(x, k \omega)=\exp (i k \omega \cdot x)$ and for $g \in L^{2}(\Omega)$ we define

$$
\varphi_{0}^{g}(x, k)=\int_{\Omega} \varphi_{0}(x, k \omega) g(\omega) d \omega .
$$

LEMMA 2.3. For any $t>\frac{1}{2}$ and any integer $m \geqq 0$

$$
\varphi_{0}^{g}(\cdot, k) \in H^{m,-t} \cap C^{\infty}\left(\mathrm{R}^{3}\right) .
$$

Let $K \subset R_{+}$be a compact set. There exists a constant $C=C(m, t, K)$ such that

$$
\left\|\varphi_{0}^{g}(\cdot, k)\right\|_{m,-t} \leqq C\|g\|_{\Omega}
$$

for all $g \in L^{2}(\Omega)$ and all $k \in K$. Moreover, $k \mapsto \varphi_{0}^{g}(\cdot, k)$ is continuous $\mathbf{R}_{+} \rightarrow H^{m,-t}$, equicontinuous for $g$ in a bounded subset of $L^{2}(\Omega)$.

For a proof, see [2]. The last statement follows from the proof given in [2]. We note that

$$
\varphi_{0}^{g}(x, k)=(2 \pi)^{\frac{3}{2}}\left(\mathscr{F}^{-1} \gamma(k)^{*} g\right)(x)
$$

where $\gamma(k)^{*} \in \mathscr{B}\left(L^{2}(\Omega), H^{-t, 0}\right)$.

It is a consequence of Lemma 2.2 that $H$ has no singular continuous spectrum. We denote by $L_{p}^{2}$ the closed subspace spanned by the eigenvectors of $H$ and by $L_{\mathrm{ac}}^{2}$ the subspace of absolute continuity of $H$. Then $L^{2}=L_{p}^{2} \oplus L_{\mathrm{ac}}^{2}$. The proof is analogous to the proof of theorem 6.1 in [2].

\section{Existence of generalized eigenfunctions.}

We denote by $x$ and $\xi$ the variables in configuration space and momentum space. We always use polar coordinates in momentum space: $\xi=k \omega, \omega \in \Omega$. We use the notation

$$
e=\left\{k \in \mathbf{R}_{+} \mid k^{2} \in e_{+}(H)\right\} .
$$

THEOREM 3.1. There exist two families 


$$
\varphi_{ \pm}(x, k, \omega), \quad x \in \mathbf{R}^{3}, k \in \mathbf{R}_{+} \backslash e, \omega \in \Omega
$$

of generalized eigenfunctions for $H$ with the following properties

(a) $\varphi_{ \pm} \in L_{\text {loc }}^{2}\left(\mathbf{R}^{3} \times R_{+} \backslash e \times \Omega ; d x \times k^{2} d k \times d \omega\right)$

(b) $(x, k) \mapsto \varphi_{ \pm}(x, k, \cdot)$ is continuous $\mathbf{R}^{3} \times \mathbf{R}_{+} \backslash e \rightarrow L^{2}(\Omega)$

(c) for $g \in L^{2}(\Omega)$ we define $\varphi_{ \pm}^{g}(x, k)=\int_{\Omega} \varphi_{ \pm}(x, k, \omega) g(\omega) d \omega$. Then $\varphi_{ \pm}^{g}(\cdot, k) \in H^{2,-s} \cap C^{\theta,-s}, k \in \mathbf{R}_{+} \backslash e$, where $s$ is the constant from the assumptions on $V, 0<\theta<\frac{1}{2}$, and furthermore

$$
\begin{gathered}
\left(H_{0}+V-k^{2}\right) \varphi_{ \pm}^{g}(\cdot, k)=0 \quad \text { for } k \in \mathrm{R}_{+} \backslash e, g \in L^{2}(\Omega) \\
\varphi_{ \pm}^{g}(\cdot, k)=\left(1-R\left(k^{2} \mp i 0\right) V\right) \varphi_{0}^{g}(\cdot, k) \quad \text { for } k \in \mathbf{R}_{+} \backslash e, g \in L^{2}(\Omega)
\end{gathered}
$$

Remark. When $V$ is multiplicative the families $\varphi_{ \pm}(x, k, \omega)$ are measuretheoretically equivalent to the generalized eigenfunctions constructed in [2]. They are unique in the same sense, see [2].

Proof. According to lemma 2.3, $\varphi_{0}^{g}(\cdot, k) \in H^{2,-s}$ for any $g \in L^{2}(\Omega)$. Thus for $k \in \mathbf{R}_{+} \backslash e$,

$$
\left(1-R\left(k^{2} \mp i 0\right) V\right) \varphi_{0}^{g}(\cdot, k) \in H^{2,-s} .
$$

From lemmas 2.1, 2.2, 2.3 and the definition of $H^{2,-s}$ follow for a fixed $x \in \mathrm{R}^{3}$ the estimate

$$
\left|\left(\left(1-R\left(k^{2} \mp i 0\right) V\right) \varphi_{0}^{g}(\cdot, k)\right)(x)\right| \leqq C\|g\|_{\Omega}
$$

for all $g \in L^{2}(\Omega)$ with $C$ independent of $g$. Hence

$$
g \mapsto\left(\left(1-R\left(k^{2} \mp i 0\right) V\right) \varphi_{0}^{g}(\cdot, k)\right)(x)
$$

is a bounded linear functional on $L^{2}(\Omega)$ and thus there exists $\varphi_{ \pm}(x, k, \cdot) \in L^{2}(\Omega)$ such that for all $g \in L^{2}(\Omega)$

$$
\int_{\Omega} \dot{\varphi}_{ \pm}(x, k, \omega) g(\omega) d \omega=\left(\left(1-R\left(k^{2} \mp i 0\right) V\right) \varphi_{0}^{g}(\cdot, k)\right)(x) .
$$

By lemmas 2.1, 2.2, 2.3 and some elementary estimates together with

$$
\left\|\varphi_{ \pm}(x, k, \cdot)-\varphi_{ \pm}\left(x^{\prime}, k^{\prime}, \cdot\right)\right\|_{\Omega}=\sup _{\substack{g \in L^{2}(\Omega) \\ g \|_{\Omega}=1}}\left|\int_{\Omega}\left(\varphi_{ \pm}(x, k, \omega)-\varphi_{ \pm}\left(x^{\prime}, k^{\prime}, \omega\right)\right) g(\omega) d \omega\right|
$$

the two maps $(x, k) \mapsto \varphi_{ \pm}(x, k, \cdot)$ are continuous $\mathbf{R}^{3} \times \mathbf{R}_{+} \backslash e \rightarrow L^{2}(\Omega)$. Thus (b) is proved. (a) follows easily from (b). Now it is a consequence of our definitions that for $k \in R_{+} \backslash e$ 


$$
\varphi_{ \pm}^{g}(x, k)=\left(\left(1-R\left(k^{2} \mp i 0\right) V\right) \varphi_{0}^{g}(\cdot, k)\right)(x) .
$$

Thus $\left(1+|\cdot|^{2}\right)^{-s / 2} \varphi_{ \pm}^{g}(\cdot, k) \in H^{2,0}$ and from Lemma 2.1 we get $\varphi_{ \pm}^{g}(\cdot, \mathrm{k}) \in \mathrm{C}^{\theta,-s}$ for any $0<\theta<\frac{1}{2}$. Equation (3.1) is proved as follows. For $g \in L^{2}(\Omega), k \in R_{+} \backslash e$ we have

$$
\left(H_{0}+V-k^{2}\right)\left(1-R\left(k^{2} \mp i \varepsilon\right) V\right) \varphi_{0}^{g}(\cdot, k)= \pm i_{\varepsilon} R\left(k^{2} \mp i \varepsilon\right) V \varphi_{0}^{g}(\cdot, k)
$$

since $H_{0}+V-k^{2}$ is a bounded linear operator from $H^{2,-s}$ to $H^{0,-s}$. Using lemmas 2.3 and 2.2 we can let $\varepsilon$ tend to zero and thus prove (3.1).

Remark. The proof depends on the dimension of the space being 3. For the case $L^{2}\left(R^{n}\right)$ one must require instead of (1.2) that

$$
V \in \mathscr{C}\left(H^{m,-s}\left(\mathrm{R}^{n}\right), H^{m-2, s}\left(\mathrm{R}^{n}\right)\right)
$$

for some $m>n / 2$. The above results can be established under this assumption. For $V$ multiplication by $v(x)(3.3)$ implies $v \in H_{\mathrm{loc}}^{m-2}\left(\mathrm{R}^{n}\right)$ and is thus stronger than Agmon's assumption for $n>3$.

THEOREM 3.2. Let $\varphi_{ \pm}$be the two families of generalized eigenfunctions from Theorem 3.1.

(a) If $s>\frac{3}{2}$ then $\varphi_{ \pm}(x, k, \omega)$ are jointly continuous in $(x, k, \omega)$. For fixed $k \in \mathbf{R}_{+} \backslash e, \omega \in \Omega$,

$$
\varphi_{ \pm}(\cdot, k, \omega) \in H^{2,-s} \cap C^{\theta,-s}, \quad 0<\theta<\frac{1}{2},
$$

and

$$
\left(H_{0}+V-k^{2}\right) \varphi_{ \pm}(\cdot, k, \omega)=0 .
$$

(b) If $s>1$ and $V$ has an extension to $H^{2,-s-\frac{1}{2}}$ such that

$$
V \in \mathscr{C}\left(H^{2,-s-\frac{1}{2}}, H^{0, s-\frac{1}{2}}\right)
$$

then $\varphi_{ \pm}(x, k, \omega)$ are jointly continuous in $(x, k, \omega)$. For fixed $k \in \mathbf{R}_{+} \backslash e, \omega \in \Omega$,

$$
\varphi_{ \pm}(\cdot, k, \omega) \in H^{2,-s-\frac{1}{2}} \cap C^{\theta,-s-\frac{1}{2}}, \quad 0<\theta<\frac{1}{2},
$$

and

$$
\left(H_{0}+V-k^{2}\right) \varphi_{ \pm}(\cdot, k, \omega)=0 .
$$

Remark. These results are well known for multiplicative $V$. See $[2$, p. 170] and the references given there. We note that for a multiplicative $V(3.5)$ is implied by assumption (1.1).

Proof. We prove (a), the proof of (b) is analogous. $s>\frac{3}{2}$ implies that 
$\varphi_{0}(\cdot, k \omega) \in H^{2,-s}$ and that $(k, \omega) \rightarrow \varphi_{0}(\cdot, k \omega)$ is a continuous map from $R_{+} \backslash e$ $\times \Omega$ to $H^{2,-s}$. It is easy to see that (after correcting $\varphi_{ \pm}$on a null set) we have

$$
\varphi_{ \pm}(x, k, \omega)=\left(\left(1-R\left(k^{2} \mp i 0\right) V\right) \varphi_{0}(\cdot, k, \omega)\right)(x) .
$$

Now the regularity results follow from lemma 2.1, 2.2 and the above mentioned continuity property of $\varphi_{0}(\cdot, k \omega)$. Equation (3.4) can be proved in the same manner as (3.1) was proved.

\section{The expansion theorem.}

Instead of giving a selfcontained proof of the expansion theorem we use the results from [4].

THEOREM 4.1. Let $\varphi_{ \pm}$denote the two families of generalized eigenfunctions from theorem 3.1. There exist two bounded linear operators $\mathscr{F}_{ \pm}$on $L^{2}$ with the following properties:

(a) $\mathscr{F}_{ \pm}$are partial isometries with initial space $L_{\mathrm{ac}}^{2}$ and final space $L^{2}$.

(b) For any $f \in L^{2}$

$$
\left(\mathscr{F}_{ \pm} f\right)(k \omega)=\lim _{N \rightarrow \infty}(2 \pi)^{-\frac{3}{2}} \int_{|x|<N} f(x) \overline{\varphi_{ \pm}(x, k, \omega)} d x
$$

and

$$
\left(\mathscr{F}_{ \pm}^{*} f\right)(x)=\lim _{j \rightarrow \infty}(2 \pi)^{-\frac{3}{2}} \int_{I_{j}} \int_{\Omega} f(k \omega) \varphi_{ \pm}(x, k, \omega) d \omega k^{2} d k
$$

where $I_{j}$ is an increasing sequence of compact sets such that $U_{j} I_{j}=R_{+} \backslash$.

(c) Let $P_{\mathrm{ac}}$ denote the projection of $L^{2}$ onto $L_{\mathrm{ac}}^{2}$. Then

$$
\left(P_{\mathrm{ac}} H\right) f=\left(\mathscr{F}_{ \pm}^{*} M_{|\cdot|^{2}} \mathscr{F}_{ \pm}\right) f \quad \text { for all } f \in D(H),
$$

where $M_{|\cdot|^{2}}$ denotes multiplication by $k^{2}$.

(d) For $f \in H^{0, s}, k \in \mathbf{R}_{+} \backslash e$

$$
\left(\mathscr{F}_{ \pm} f\right)(k \omega)=\left(\gamma(k)\left(\mathscr{F}\left(1-V R\left(k^{2} \pm i 0\right)\right) f\right)\right)(\omega) .
$$

Proof. For $f \in H^{0, s}$ we define

$$
\left(F_{ \pm} f\right)(k \omega)=\gamma(k)\left(\mathscr{F}\left(1-V R\left(k^{2} \pm i 0\right)\right) f\right)(\omega) .
$$

It is proved in [4, section 5.5] that $F_{ \pm}$can be extended to a bounded linear operator on $L^{2}$ with the properties (a) and (c). We denote by $L_{c}^{2}$ the $L^{2}$-functions with compact support. For $f \in L_{c}^{2}, h \in L^{2}(\Omega)$ we have 


$$
\begin{aligned}
& \int_{\Omega} \overline{h(\omega)}\left(\mathscr{F}\left(1-V R\left(k^{2} \pm i 0\right)\right) f\right)(k \omega) d \omega \\
& =\int_{\Omega} \overline{h(\omega)} \lim _{N \rightarrow \infty}(2 \pi)^{-\frac{3}{2}} \int_{|x|<N}\left(\left(1-V R\left(k^{2} \pm i 0\right)\right) f\right)(x) \varphi_{0}(x,-k \omega) d x d \omega \\
& =\lim _{N \rightarrow \infty}(2 \pi)^{-\frac{3}{2}} \overline{h(\omega)} \int_{|x|<N}\left(\left(1-V R\left(k^{2} \pm i 0\right)\right) f\right)(x) \varphi_{0}(x,-k \omega) d x d \omega \\
& =\lim _{N \rightarrow \infty}(2 \pi)^{-\frac{3}{2}} \int_{|x|<N} \overline{\left(\left(1-V R\left(k^{2} \pm i 0\right)\right) f\right)(x)} \overline{\int_{\Omega} h(\omega) \varphi_{0}(x, k \omega) d \omega d x} \\
& =(2 \pi)^{-\frac{3}{2}} \int_{R^{3}} f(x) \overline{\left(1-R\left(k^{2} \mp i 0\right) V\right) \varphi_{0}^{h}(\cdot, k)} d x \\
& =(2 \pi)^{-\frac{3}{2}} \int_{R^{3}} f(x) \overline{\int_{\Omega}} \overline{\varphi_{ \pm}(x, k, \omega) h(\omega) d \omega d x} \\
& =\int_{\Omega} \overline{h(\omega)(2 \pi)^{-\frac{3}{2}}} \int_{R^{3}} f(x) \overline{\varphi_{ \pm}(x, k, \omega)} d x d \omega .
\end{aligned}
$$

The exchange of the limit and the integration above is legitimate, because

$$
\begin{aligned}
& \left(\mathscr{F}\left(1-V R\left(k^{2} \pm i 0\right)\right) f\right)(k \omega) \\
& \quad=(2 \pi)^{-\frac{3}{2}} \lim _{N \rightarrow \infty} \int_{|x|<N}\left(\left(1-V R\left(k^{2} \pm i 0\right)\right) f\right)(x) e^{-i x \cdot k \omega} d x
\end{aligned}
$$

the convergence takes place in $H^{s, 0}$, as $\mathscr{F}$ maps $H^{0, s}$ onto $H^{s, 0}$. We have also used the continuity of the trace operator and the inner product on $L^{2}(\Omega)$. Furthermore we have used the fact that

$$
\int_{\mathrm{R}^{3}} f(x) \overline{\left(R\left(k^{2} \mp i 0\right) V g\right)(x)} d x=\int_{R^{3}}\left(V R\left(k^{2} \pm i 0\right) f\right)(x) \overline{g(x)} d x
$$

for $f \in H^{0, s}, g \in H^{2,-s}$, which is a consequence of the assumptions on $V$ and lemma 2.2. We have also used Fubini's theorem twice. The first use is easy to justify, the second use is justified by the following estimate

$$
\begin{aligned}
\int_{R^{3}} \int_{\Omega}\left|\varphi_{ \pm}(x, k, \omega) h(\omega) f(x)\right| d \omega d x & \\
& \leqq\|h\|_{\Omega}\|f\|_{L^{1}} \sup _{x \in \operatorname{supp}(f)}\left\|\varphi_{ \pm}(x, k \cdot)\right\|_{\Omega}<\infty .
\end{aligned}
$$

It follows from theorem 3.1(a) that for $f \in L_{c}^{2}$ we can define

$$
\left(\mathscr{F}_{ \pm} f\right)(k \omega)=(2 \pi)^{-\frac{3}{2}} \int_{\mathrm{R}^{3}} f(x) \overline{\varphi_{ \pm}(x, k, \omega)} d x
$$


and that $\mathscr{F}_{ \pm} f$ belongs to $L_{\mathrm{loc}}^{2}\left(\mathrm{R}_{+} \backslash e \times \Omega ; k^{2} d k \times d \omega\right)$. We see that for each $k \in \mathbf{R}_{+} \backslash e$

$$
\left(\mathscr{F}_{ \pm} f\right)(k \cdot)=\left(F_{ \pm} f\right)(k \cdot) \quad \text { in } L^{2}(\Omega) .
$$

$\left(F_{ \pm} f\right)(k \cdot)$ is continuous in $k$ with values in $L^{2}(\Omega)$, so we get

$$
\mathscr{F} f_{ \pm}=F_{ \pm} f, \quad f \in L_{c}^{2} .
$$

From the properties of $F_{ \pm}$stated above follow that $\mathscr{F}_{ \pm}$have unique extensions, also denoted $\mathscr{F}_{ \pm}$, to bounded operators on $L^{2}$ with the properties (a) and (c). (d) follows from (4.3) and the density of $L_{c}^{2}$ in $H^{0, s}$. Let $\chi_{N}$ denote the characteristic function of $\{x|| x \mid<N\}$. Then for any $f \in L^{2}$

$$
\begin{aligned}
\left(\mathscr{F}_{ \pm} f\right)(k \omega) & =\lim _{N \rightarrow \infty} \mathscr{F}_{ \pm}\left(\chi_{N} f\right)(k \omega) \\
& =\lim _{N \rightarrow \infty}(2 \pi)^{-\frac{3}{2}} \int_{|x|<N} f(x) \overline{\varphi_{ \pm}(x, k, \omega)} d x
\end{aligned}
$$

(convergence in $L^{2}$ ) and (4.1) is proved. It follows from theorem 3.1(a) and Fubini's theorem that for $f \in L_{c}^{2}, \operatorname{supp}(f) \leqq\left\{k \omega \mid k \in \mathrm{R}_{+} \backslash e, \omega \in \Omega\right\}$

$$
\left(\mathscr{F}_{ \pm}^{*} f\right)(x)=(2 \pi)^{-\frac{3}{2}} \int_{\mathrm{R}_{+} \backslash e} \int_{\Omega} f(k \omega) \varphi_{ \pm}(x, k, \omega) d \omega k^{2} d k .
$$

Now the proof of (4.2) is analogous to the proof of (4.1).

\section{Representation of the scattering matrix.}

We refer to [4] for a discussion of scattering theory. We summarize some results from [4] in the following theorem:

Theorem 5.1. (Kuroda). Define $W_{ \pm}=\mathscr{F}_{ \pm}^{*} \mathscr{F}$. Then

$$
W_{ \pm}=s-\lim _{t \rightarrow \pm \infty} e^{i t H} e^{-i t H_{0}}
$$

and range $\left(W_{ \pm}\right)=L_{\mathrm{ac}}^{2}$, that is the wave operators exist and are complete. The unitary scattering operator $S=W_{+}^{*} W_{-}$has a representation

$$
(\mathscr{F} S f)(k \omega)=S(k)(\mathscr{F} f)(k \cdot)(\omega)
$$

where $S(k), k \in R_{+} \backslash e$ is a family of unitary operators on $L^{2}(\Omega)$, depending continuously on $k$ in the operator norm on $L^{2}(\Omega)$. $S(k)$ has the following representation

$$
S(k)=1-\pi i k \gamma(k) \mathscr{F}\left[V-V R\left(k^{2}+i 0\right) V\right] \mathscr{F}^{-1} \gamma(k)^{*} .
$$


Remark. It follows from the remark after lemma 2.3 that $\mathscr{F}^{-1} \gamma(k)^{*}$ maps $L^{2}(\Omega)$ into $H^{2,-s}$ boundedly.

THEOREM 5.2. $S(k)-1$ has the kernel $t\left(k ; \omega, \omega^{\prime}\right)$, symbolically given by

$$
t\left(k ; \omega, \omega^{\prime}\right) \sim-\frac{i}{8 \pi^{2}} k \int_{\mathrm{R}^{3}} \varphi_{0}(x,-k \omega) V \varphi_{-}\left(x, k, \omega^{\prime}\right) d x .
$$

This is to be interpreted as follows. For $f, g \in L^{2}(\Omega)$ and $k \in \mathrm{R}_{+} \backslash e$

$$
(f,(S(k)-1) g)_{\Omega}=-\frac{i}{8 \pi^{2}} k \int_{\mathrm{R}^{3}} \overline{\varphi_{0}^{f}(x, k)} V \varphi_{-}^{g}(x, k) d x .
$$

REMARK. (5.2) is the representation of the scattering amplitude in terms of generalized eigenfunctions formally derived in physics textbooks, see for instance [6].

Proof. From (5.1) follows for $k \in \mathrm{R}_{+} \backslash e, f, g \in L^{2}(\Omega)$

$$
\begin{aligned}
& (f,(S(k)-1) g)_{\Omega} \\
& =-\pi i k\left(f, \gamma(k) \mathscr{F}\left[V-V R\left(k^{2}+i 0\right) V\right] \mathscr{F}^{-1} \gamma(k)^{*} g\right)_{\Omega} \\
& =-\pi i k\left(\mathscr{F}^{-1} \gamma(k)^{*} f, V\left(1-R\left(k^{2}+i 0\right) \bigvee\right)^{-1} \gamma(k)^{*} g\right) \\
& =-\frac{i k}{8 \pi^{2}}\left(\varphi_{0}^{f}(\cdot, k), V \varphi_{-}^{g}(\cdot, k)\right)
\end{aligned}
$$

where we have used the remark after lemma 2.3 and (3.2).

\section{REFERENCES}

1. S. Agmon, On kernels, eigenvalues, and eigenfunctions of operators related to elliptic problems, Comm. Pure Appl. Math. 18 (1965), 627-663.

2. S. Agmon, Spectral properties of Schrödinger operators and scattering theory, Ann. Scuola Norm. Sup. Pisa, C1. Sci. 2 (1975), 151-218.

3. E. Balslev, The singular spectrum of elliptic differential operators in $L^{p}\left(\mathbf{R}^{n}\right)$, Math. Scand. 19 (1966), 193-210.

4. S. T. Kuroda, Lecture notes on scattering theory, Aarhus University, 1976.

5. J. L. Lions and E. Magenes, Non-homogeneous boundary value problems and applications I (Grundlehren Math. Wissensch. 81), Springer-Verlag, Berlin - Heidelberg - New York, 1972.

6. R. G. Newton, Scattering theory for Waves and particles, McGraw-Hill, New York, 1966.

7. S. L. Sobolev, Applications of functional analysis in mathematical physics (Translations of Math. Monographs 7), Amer. Math. Soc., Providence, 1963. 
8. O. Yamada, Eigenfunction expansions and scattering theory for Dirac operators, Publ. Res. Inst. Math. Sci. 11 (1976), 651-689.

\author{
DEPARTMENT OF MATHEMATICS \\ UNIVERSITY OF CALIFORNIA \\ BERKELEY, CALIFORNIA 94720 \\ U.S.A.
}

AND

MATEMATISK INSTITUT

AARHUS UNIVERSITET

NY MUNKEGADE

DK 8000 AARHUS C

DENMARK 